Commun. Fac. Sci. Univ. Ank. Series A1

V. 46. pp. 93-101 (1997)

\title{
PROPERTIES OF 2-DIMENSIONAL SPACE-LIKE RULED SURFACES IN THE MINKOWSKI SPACE $\mathbf{R}_{1}{ }^{\mathbf{n}}$
}

İsmail AYDEMİR* - Murat TOSUN** - Nuri KURUOĞLU*

* Department of Mathematics, Faculty of Educations, Ondokuz Mayls University, Samsun, TURKEY

** Department of Mathematics, Faculty of Arts and Sciences, Sakarya University, Sakarya, TURKEY

(Received May 26, 1997; Revised Dec. 6, 1997; Accepted Dec. 9, 1997)

\section{ABSTRACT}

In this paper we find new characteristic properties for 2-dimensional ruled surface $M$ in $\mathbb{R}_{1}{ }^{n}$ and give the sufficient and necessary conditions for which the space-like ruled surface $M$ is to be total geodesic. In addition, some characterisation which is the well-known for the ruled surfaces in the Euclidean 3-space was generalized for the space-like ruled surfaces in $\mathbb{R}_{1}{ }^{n}$.

\section{INTRODUCTION}

We shall assume throughout this paper that all manifolds, maps vector fields, etc... are differentiable of class $\mathrm{C}^{\infty}$. Consider a general submanifold $M$ of the Minkowski space $\mathbb{R}_{1}{ }^{n}$. Suppose that, $\bar{D}$ is the Levi-Civita connection of Minkowski space $\mathbb{R}_{1}{ }^{n}$, while $D$ is the Levi-Civita connection of Semi Riemann manifold $M$. If $X$ and $Y$ are the vector fields of $M$ and if $V$ is second fundamental form of $M$, we have by decomposing $\mathrm{D}_{\mathrm{X}} \mathrm{Y}$ in a tangential and normal component.

$$
\bar{D}_{X} Y=D_{X} Y+V(X, Y)
$$

The equation (1.1) is called Gauss equation, [1].

If $\xi$ is any normal vector filed on $M$, we find the Weingarten equation by decomposing $\overline{\mathrm{D}}_{\mathrm{X}} \xi$ in a tangential and normal component

$$
\bar{D}_{x} \xi=-A_{\xi}+D_{x}^{\perp} \xi
$$


$A_{\xi}$ determines at each point a self-adjoint lineer map and $D^{\perp}$ is a metric connection in the normal bundle $\chi^{\perp}(M)$. We use the same notation $A_{\xi}$ for the linear map and the matrix of the lineer map, [1].

A normal vector field $\xi$ is called parallel in the normal bundle $\chi^{\perp}$ (M) if we have $D_{X}^{\perp} \xi=0$ for each vector $X$. If $\eta$ is a normal unit vector at the point $p \in M$, then

$$
\mathrm{G}(\mathrm{p}, \eta)=\operatorname{det} \mathrm{A}_{\eta}
$$

is the Lipschitz-Killing curvature of $M$ at $p$ in direction $\eta,[2]$.

Suppose that $\mathrm{X}$ and $\mathrm{Y}$ are vector fields on $\mathrm{M}$, while $\xi$ is a normal vector field on $\chi^{\perp}(M)$. If the standart metric tensor of $\mathbb{R}_{1}{ }^{n}$ is doneted by $<>$ then we have

$$
\left\langle\bar{D}_{\mathrm{X}} \mathrm{Y} \xi\right\rangle=\langle\mathrm{V}(\mathrm{X}, \mathrm{Y}) \xi\rangle
$$

and

$$
\left\langle\bar{D}_{X} Y \xi\right\rangle=\left\langle A_{\xi}(X), Y\right\rangle
$$

From the above equations we obtain

$$
\langle\mathrm{V}(\mathrm{X}, \mathrm{Y}), \xi\rangle=\left\langle\mathrm{A}_{\xi}(\mathrm{X}), \mathrm{Y}\right\rangle
$$

If $\xi_{1}, \xi_{2}, \ldots, \xi_{\mathrm{n}-2}$ constitute an orthonormal base field of the normal bundle $\chi^{\perp}(\mathrm{M})$, then we set

$$
\left\langle\mathrm{V}(\mathrm{X}, \mathrm{Y}), \xi_{\mathrm{j}}\right)=\mathrm{V}_{\mathrm{j}}(\mathrm{X}, \mathrm{Y})
$$

or

$$
\mathrm{V}(\mathrm{X}, \mathrm{Y})=\sum_{\mathrm{j}=1}^{\mathrm{n}-2} \mathrm{~V}_{\mathrm{j}}(\mathrm{X}, \mathrm{Y}) \xi_{\mathrm{j}}
$$

The mean curvature vector $H$ of $M$ at the point $p$ is given by

$$
\mathrm{H}=\sum_{\mathrm{j}=1}^{\mathrm{n}-2} \frac{\mathrm{tr} \mathrm{A}_{\xi_{\mathrm{j}}}}{2} \xi_{\mathrm{j}}
$$

$\|\mathrm{H}\|$ is the mean curvature. If $\mathrm{H}=0$ at each point $\mathrm{p}$ of $\mathrm{M}$, then $\mathrm{M}$ is said to be minimal, [1]. 


\section{2-DIMENSIONAL SPACE-LIKE RULED SURFACES IN $\mathbf{R}_{1}{ }^{\mathbf{n}}$}

Let $\alpha$ be a space-like curve and e(s) be a space-like unit vector on the generators in $\mathbb{R}_{1}{ }^{n}$. If the space-like base curve $\alpha$ is an orthogonal trajectory of the generators then we get a 2-dimensional ruled surface $M$. This ruled surface is called 2-dimensional space-like ruled surface and represented by

$$
\psi(\mathrm{s}, \mathrm{v})=\mathrm{a}(\mathrm{s})+\mathrm{v} \mathrm{e}(\mathrm{s}) .
$$

Definition 2.1: Let $M$ be 2-dimensional space-like ruled surface in $\mathbb{R}_{1}{ }^{n}$ and $\mathrm{V}$ be second fundamental form of $\mathrm{M}$. If $\mathrm{V}(\mathrm{X}, \mathrm{X})=0$ for all $\mathrm{X}$ $\in \chi(M)$ then $\mathrm{X}$ is called an asymptotic vector field on $\mathrm{M}$.

Theorem 2.1: Let $M$ be 2-dimensional space-like ruled surface in $\mathbb{R}_{1}{ }^{n}$. Then the generators of $M$ are asymptotics and geodesics of $M$.

Proof: Since the generators are the geodesics of $\mathbb{R}_{1}{ }^{n}$, we have

$$
\overline{\mathrm{D}}_{\mathrm{e}} \mathrm{e}=0 \text {. }
$$

If we set this in the Gauss equation, we get

$$
D_{e} e+V(e, e)=0 \text { or } D_{e} e=-V(e, e) \text {. }
$$

Since $D_{e} e \in x(M)$ and $V(e, e) \in \chi^{\perp}(M)$ we get $D_{e} e=0$ and $V(e, e)=0$.

Therefore the generators of $M$ are the asymptotics and geodesics of $M$.

Suppose that $\left\{\mathrm{e}_{1}, \mathrm{e}\right\}$ is an orthonormal base field of the tangential bundle $\chi(\mathrm{M})$ and $\left\{\xi_{1}, \xi_{2}, \ldots, \xi_{\mathrm{n}-2}\right\}$ is an orthonormal bundle $\chi^{\perp}(\mathrm{M})$. Then we have the following equations.

$$
\begin{array}{ll}
\bar{D}_{e} \xi_{j}=a_{11}^{j} e+a_{12}^{j} e_{1}+\sum_{i=1}^{n-2} b_{1 i}^{j} \xi_{i}, & 1 \leq j \leq n-2 \\
\bar{D}_{e_{1}} \xi_{j}=a_{21}^{j} e+a_{22}^{j} e_{1}+\sum_{i=1}^{n} b_{2 i}^{j} \xi_{i}, & 1 \leq j \leq n-2
\end{array}
$$

From these eqations we observe that

$$
a_{21}^{j}=-a_{12}^{j}, \quad a_{11}^{j}=0, \quad 1 \leq j \leq n
$$

and 


$$
A_{\xi_{j}}=-\left[\begin{array}{ll}
0 & a_{12}^{j} \\
a_{12}^{j} & a_{22}^{j}
\end{array}\right] .
$$

Then we have the following corollary.

Corollary 2.1: The matrix $A_{\xi_{j}}$ is corresponding to the shape operator of $M$ and $A_{\xi_{j}}$ is a symmetric matrix in the sense of Lorentz.

Corollary 2.2: The Lipschitz-Killing curvature at $p \in M$ in the direction of $\xi_{\mathrm{j}}$ is given by

$$
G\left(p, \xi_{j}\right)=-\left(a_{12}\right)^{2} \text {. }
$$

From (2.1) we have

$$
a_{12}^{j}=\left\langle\bar{D}_{e} \xi_{j}, e_{1}\right\rangle=-\left\langle\xi_{j}, \bar{D}_{e} e_{1}\right\rangle
$$

and

$$
\left\langle\bar{D}_{e} e_{1} e\right)=-\left\langle e_{1}, \bar{D}_{e} e\right)=0
$$

while

$$
\left\langle\bar{D}_{e} e_{1} e_{1}\right\rangle=-\left\langle e_{1}, \bar{D}_{e} e_{1}\right\rangle=0
$$

From (2.4) and (2.5) we observe that $\vec{D}_{e} e_{1} \in \chi^{\perp}(M)$ or $\bar{D}_{e} e_{1}=V\left(e, e_{1}\right)$. Because of (2.3) we have

$$
\begin{aligned}
& \overline{\mathrm{D}}_{\mathrm{e}} \mathrm{e}_{1}=\mathrm{V}\left(\mathrm{e} \mathrm{e}_{1}\right)=\sum_{\mathrm{j}=1}^{\mathrm{n}-2} \varepsilon_{\mathrm{j}}\left\langle\xi_{\mathrm{i}}, \overline{\mathrm{D}}_{\mathrm{e}} \mathrm{e}\right) \xi_{\mathrm{j}}=-\sum_{\mathrm{j}=1}^{\mathrm{n}-2} \varepsilon_{\mathrm{j}} \mathrm{a}_{12}^{\mathrm{j}} \xi_{\mathrm{j}} \\
& \varepsilon_{\mathrm{j}}=\left\langle\xi_{\mathrm{j}}, \xi_{\mathrm{j}}\right\rangle=\left\{\begin{array}{l}
-1, \xi_{\mathrm{j}} \text { time-like } \\
1, \xi_{\mathrm{j}} \text { space-like . }
\end{array}\right.
\end{aligned}
$$

Because of (1.4) and (2.1) we find

$$
a_{2}^{j}=\left\langle D_{e_{1}} \xi_{j}, e_{1}\right\rangle=-\left\langle A_{\xi_{j}}\left(e_{1}\right), e_{1}\right\rangle=-\left\langle\left(e_{1}, e_{1}\right), \xi_{j}\right\rangle
$$

and

$$
\operatorname{tr} A_{\xi_{j}}=-a_{22}^{j}=\left\langle\left(e_{1}, e_{1}\right), \xi_{j}\right\rangle, \quad 1 \leq j \leq n-2 .
$$


Theorem 2.2: Let $M$ be 2-dimensional space-like ruled surface in $\mathbb{R}_{1}{ }^{n}$ and $\left\{\mathrm{e}_{1}, e\right\}$ be the orthonormal base field of the tangential bundle $\chi(M)$. Then the Gauss curvature $G$ can be given as follows

$$
\mathrm{G}=\left\langle\overline{\mathrm{D}}_{\mathrm{e}} \mathrm{e}_{1}, \overline{\mathrm{D}}_{\mathrm{e}} \mathrm{e}_{1}\right\rangle \text {. }
$$

Proof: Let $R$ be the Riemannian curvature tensor field of $M$. In this case we get

$$
\mathrm{G}=\left\langle\mathrm{R}\left(\mathrm{e}_{1}, \mathrm{e}\right) \mathrm{e}, \mathrm{e}_{1}\right\rangle,[3] \text {. }
$$

By combining (2.9) and $V(e, e)=0$ we are faced with

$$
\mathrm{G}=\left\langle\mathrm{V}\left(\mathrm{e}, \mathrm{e}_{1}\right), \mathrm{V}\left(\mathrm{e}, \mathrm{e}_{1}\right)\right\rangle
$$

or

$$
G=\left\langle\bar{D}_{e_{1}} e_{1} \bar{D}_{e} e_{1}\right\rangle
$$

From the above Theorem 2.2 Corollary 2.2 and the equation (2.6) we have the following corollaries.

Corollary 2.3: The Gauss curvature of $M$ with respect to the elements of $A_{\xi}$.

$$
\mathrm{G}=\sum_{\mathrm{j}=1}^{\mathrm{n} 2} \varepsilon_{\mathrm{j}}\left(\mathrm{a}_{12}^{\mathrm{j}}\right)^{2}
$$

Corollary 2.4: A space-like ruled surface $M$ is developable if and only if the Lipschitz-Killing curvture is zero at each point.

Theorem 2.3: Let $M$ be a 2-dimensional space-like ruled surface in $\mathbb{R}_{1}{ }^{n}$. The mean curvature of $M$ is

$\mathrm{H}=\frac{1}{2} \varepsilon_{\mathrm{j}} \mathrm{V}\left(\mathrm{e}_{1}, \mathrm{e}_{1}\right)$.

Proof: From (1.8) we know that

$$
\mathbf{H}=\sum_{j=1}^{\mathbf{n} 2} \frac{\text { tr } A_{\xi_{j}}}{2} \xi_{j} \text {. }
$$

For the matrix $A_{\xi_{j}}$ given (2.2) we find

$$
\text { tr } A_{\xi_{j}}=-a_{22}^{j}
$$


If we substitude (2.8) in (1.8) we get

$$
\mathrm{H}=\frac{1}{2} \varepsilon_{\mathrm{j}} \mathrm{V}\left(\mathrm{e}_{1}, \mathrm{e}_{1}\right) \text {. }
$$

Theorem 2.4: Let $M$ be 2-dimensional space-like ruled surface in $\mathbb{R}_{1}{ }^{n} . M$ is developable and minimal iff $M$ is total geodesic.

Proof: We assume that $M$ is developable and minimal. If $X, Y \in \chi(M)$, we have $X=a e+b e_{1}$ and $Y=c e+d e_{1}$.

Therefore we get

$$
\mathrm{V}(\mathrm{X}, \mathrm{Y})=\mathrm{ac} \mathrm{V}(\mathrm{e}, \mathrm{e})+(\mathrm{ad}+\mathrm{bc}) \mathrm{V}\left(\mathrm{e}, \mathrm{e}_{1}\right)+b d \mathrm{~V}\left(\mathrm{e}_{1}, \mathrm{e}_{1}\right) .
$$

Because of Theorem 2.1 and minimality of $M$ we have $V(e, e)=0$ and $V\left(e_{1}, e_{1}\right)=0$. Moreover, since $M$ is developable $D_{e} e_{1}=0$. Thus we can write $\mathrm{V}\left(\mathrm{e}, \mathrm{e}_{1}\right)=0$ and $\mathrm{V}(\mathrm{X}, \mathrm{Y})=0$ for all $\mathrm{X}, \mathrm{Y} \in \chi(\mathrm{M})$.

Now suppose that $\mathrm{V}(\mathrm{X}, \mathrm{Y})=0, \forall \mathrm{X}, \mathrm{Y} \in \chi(\mathrm{M})$. Then we have $\mathrm{V}$ $(\mathrm{e}, \mathrm{e})=0, \mathrm{~V}\left(\mathrm{e}, \mathrm{e}_{1}\right)=0$. Because of Theorem 2.1 we have

$$
\left\langle\bar{D}_{\mathrm{e}} \mathrm{e}_{1} \mathrm{e}\right\rangle=0 \text { and }\left\langle\overline{\mathrm{D}}_{\mathrm{e}} \mathrm{e}_{1}, \mathrm{e}_{1}\right\rangle=0 \text {. }
$$

This means that $\bar{D}_{e} e_{1}$, is a normal vector field or $\bar{D}_{e} e_{1}=V\left(e, e_{1}\right)$.

Therefore we have $\bar{D}_{e} e_{1}=0$. This implies that $M$ is developable and $V$ $\left(\mathrm{e}, \mathrm{e}_{1}\right)=0$ implies that $\mathrm{M}$ is minimal.

Let $M$ be 2-dimensional space-like ruled surface in $\mathbb{R}_{1}{ }^{n}$ and $e$ be unit space-like vector field of the generator. Then we have the following equations of covariant derivative of the orthonormal base field $\left\{e, e_{1}, \xi_{1}\right.$, $\left.\xi_{2}, \ldots \xi_{\mathrm{n}-2}\right\}$.

$$
\begin{aligned}
& \bar{D}_{e_{1}} e_{1}=c_{11} e_{1}+c_{12} e^{e+c_{13}} \xi_{1}+\ldots+c_{1 n} \xi_{n-2} \\
& \bar{D}_{e_{1}} e=c_{21} e_{1}+c_{22} e^{e+c_{23}} \xi_{1}+\ldots+c_{2 n} \xi_{n-2} \\
& \bar{D}_{e_{1}} \xi_{1}=c_{31} e_{1}+c_{32} e+c_{33} \xi_{1}+\ldots+c_{3 n} \xi_{n-2} \\
& \vdots \\
& \bar{D}_{e_{1}} \xi_{n-2}=c_{n 1} e_{1}+c_{n 2} e+c_{n 3} \xi_{1}+\ldots+c_{n n} \xi_{n-2} .
\end{aligned}
$$


If we write these equations in the matrix form we get

$\left[\begin{array}{l}\bar{D}_{e_{1}} e_{1} \\ \bar{D}_{e_{1}} e \\ \bar{D}_{e_{1}} \xi_{1} \\ \vdots \\ \bar{D}_{e_{1}} \xi_{n-2}\end{array}\right]=\left[\begin{array}{lllll}0 & c_{12} & c_{13} & \ldots & c_{1 n} \\ -c_{12} & 0 & c_{23} & \ldots & c_{2 n} \\ -\varepsilon_{i} c_{13} & -\varepsilon_{i} c_{23} & 0 & \ldots & c_{3 n} \\ \vdots & & & & \\ -\varepsilon_{i} c_{1 n} & -\varepsilon_{i} c_{2 n} & -c_{3 n} & \cdots & 0\end{array}\right]\left[\begin{array}{c}e_{1} \\ e \\ \xi_{1} \\ \vdots \\ \xi_{n-2}\end{array}\right]$.

Theorem 2.5: Let $M$ be a 2-dimensional space-like ruled surface in $\mathbb{R}_{1}{ }^{\mathrm{n}}$. $\left\{\mathrm{e}_{1}, \mathrm{e}\right\}$ be an orthonormal base field of the tangential bundle $\chi(\mathrm{M})$ and $\alpha(s)$ be an orthonormal trajectory of the generators of $M$. Then the following propositions are equivalent.

i) $\mathrm{M}$ is developable

ii) The Lipschitz-Kiling curvature

$\mathrm{G}\left(\mathrm{p}, \xi_{\mathrm{j}}\right)=0,1 \leq \mathrm{j} \leq \mathrm{n}-2$

iii) The Gauss curvature $G=0$.

iv) In the equation (2.13), $\mathrm{c}_{2 \mathrm{k}}=0,3 \leq \mathrm{k} \leq \mathrm{n}$.

v) $A_{\xi_{j}}(e)=0$

vi) $\overline{\mathrm{D}}_{\mathrm{e}_{1}} \mathrm{e} \in \chi(\mathrm{M})$.

Proof: $i \Rightarrow$ ii : We assume that $M$ is developable, since $a_{11}^{j}=0$ in (2.1), $1 \leq \mathrm{j} \leq \mathrm{n}-2$, the Lipschitz-Killing curvature at point $\mathrm{p}$ in the direction of $\xi_{\mathrm{j}}$ is given by

$$
\mathrm{G}\left(\mathrm{p}, \xi_{1}\right)=-\left(\mathrm{a}_{12}^{\mathrm{j}}(\mathrm{p})\right)^{2}=0,1 \leq \mathrm{j} \leq \mathrm{n}-2 .
$$

Because of (2.6) and since $M$ is developable we have

$\overline{\mathrm{D}}_{\mathrm{e}} \mathrm{e}_{1}=-\sum_{\mathrm{j}=1}^{\mathrm{n}-2} \varepsilon_{\mathrm{j}}\left(\mathrm{a}_{12}^{\mathrm{j}}\right) \xi_{\mathrm{j}}=0$.

So we find $G\left(p, \xi_{j}\right)=0,1 \leq j \leq n-2$.

ii $\Rightarrow$ iii : Let $G\left(p, \xi_{j}\right)=0,1 \leq j \leq n-2$. 
Since we have

$$
\mathrm{G}(\mathrm{p})=-\sum_{\mathrm{j}=1}^{\mathrm{n}-2} \mathrm{G}\left(\mathrm{p}, \xi_{\mathrm{i}}\right), \forall \mathrm{p} \in \mathrm{M}
$$

we observe that $\mathrm{G}=0, \forall \mathrm{p} \in \mathrm{M}$.

iii $\Rightarrow$ iv : Suppose that $G=0, \forall p \in M$. Then because of (2.11) we have $a_{12}^{j}=0,1 \leq j \leq n-2$. So $\bar{D}_{e_{j}} \xi_{j}$ has no component in the direction e. Hence we observe that $c_{2 k}=0.3 \leq k \leq n$, in the equation (2.13).

iv $\Rightarrow \mathrm{v}$ : Suppose that $\mathrm{c}_{2 \mathrm{k}}=0.3 \leq \mathrm{k} \leq \mathrm{n}$, in the equation (2.13). That shows that $\overline{\mathrm{D}}_{\mathrm{e}_{\mathrm{j}}} \xi_{\mathrm{j}}$ has no component in the direction e. Thus we have in the equation $(2.1), a_{12}^{j}=0,1 \leq \mathrm{j} \leq \mathrm{n}-2$.

Moreover, since $a_{11}^{j}=\left\langle\bar{D}_{e_{1}} \xi_{j} e\right\rangle=-\left\langle\xi_{j} \bar{D}_{e} e\right\rangle=0$ and becacuse of the Weingarten equation we find

$$
A_{\xi_{j}}(e)=0,1 \leq j \leq n-2 .
$$

$\mathrm{v} \Rightarrow$ vi : Let $A_{\xi_{j}}(e)=0$. Then, from the Weingarten equation, we have $a_{11}^{j}=0, a_{12}^{j}=0, \quad 1 \leq j \leq n-2$. Moreover, $\left\langle e, \xi_{j}\right\rangle=0$ implies

$$
\left\langle\bar{D}_{e_{1}}, \xi_{j}\right\rangle=-\left\langle e, \bar{D}_{e_{1}} \xi_{j}\right\rangle
$$

If we se equations 2.1 and last equations we get

$$
\left\langle\bar{D}_{e_{1}}, \xi_{j}\right\rangle=-\left\langle e, \bar{D}_{e_{1}} \xi_{j}\right\rangle=-a_{12}^{j}
$$

and

$$
\left\langle\bar{D}_{\mathrm{e}_{1}} \mathrm{e}, \xi_{\mathrm{j}}\right\rangle=0
$$

From the last equation we have

$$
\overline{\mathrm{D}}_{\mathrm{e}_{1}} \mathrm{e} \in \chi(\mathrm{M})
$$

vi $\Rightarrow \mathrm{i}$ : Let $\overline{\mathrm{D}}_{\mathrm{e}_{1}} \mathrm{e} \in \chi(\mathrm{M})$. Then from the equation (2.14), we get $\left\langle\bar{D}_{e_{1}} e, \xi_{1}\right\rangle=-a^{j}=0$. $1 \leq j \leq n-2$. On the other hand, $e\left[\left\langle e_{1}, e_{1}\right\rangle\right]=e$
$[1]$ implies that $\left\langle\bar{D}_{e} e_{1}, e_{1}\right\rangle=0$ and $e\left[\left\langle e_{1}, e\right\rangle\right]=e[0]$ implies that $\left\langle D_{e} e_{1} e\right\rangle=0$ (Since the generators are the geodesics of $\mathbb{R}_{1}{ }^{n}$, we have $\overline{\mathrm{D}}_{e} \mathrm{e}=0$ ). Thus $\overline{\mathrm{D}} \mathrm{e}_{1} \in \chi(\mathrm{M})$. 
Because of (2.6) and since $a_{12}^{j}=0 . \quad 1 \leq j \leq n-2$, we write that $\bar{D}_{e} e_{1}$ $=0$.

This means tha tangent planes of $M$ constant along the generator $\mathrm{e}$ of $M$. i.e. $M$ is developable.

Corollary 2.5: Let $M$ be a 2-dimensional space-like ruled surface in $\mathbb{R}_{1}{ }^{n}$ with a Gauss curvature beign zero. If $M$ is minimal, then $c_{s k}=0,1$ $\leq \mathrm{s} \leq 2,3 \leq \mathrm{k} \leq \mathrm{n}$, in the (2.13).

Proof: Let $M$ be minimal. Then from the eqation (2.12) we have $V$ $\left(e_{1}, e_{1}\right)=0$. If this result is set in the Gauss equation, we find

$$
\overline{\mathrm{D}}_{\mathrm{e}_{1}} \mathrm{e}_{1}=\mathrm{D}_{\mathrm{e}_{1}} \mathrm{e}_{1} \text {. }
$$

This means that $\overline{\mathrm{D}}_{\mathrm{e}_{1}} \mathrm{e}_{1}$ has no component in $\chi^{\perp}(\mathrm{M})$. Therefore we have

$$
\mathrm{C}_{1 \mathrm{k}}=0,3 \leq \mathrm{k} \leq \mathrm{n} \text {. (2.15) }
$$

in the equation (2.13). On the other hand, since $G=0$, by hypothesis, and from the Theorem 2.5 we know that $C_{2 k}=0.3 \leq k \leq n$. If we consider this together with $(2.15)$ we observe that $C_{s k}=0,1 \leq s \leq 2,3$ $\leq \mathbf{k} \leq \mathbf{n}$.

\section{REFERENCES}

[1] CHEN B.Y., Geometri of Submanifolds, Marcel Dekker, New York 1973.

[2] HOUH, C.S., Surfaces with Maximal Lipschitz-Killing Curvature in the Direction of Mean Curvature Vector, Proc. Amer. Math. Soc. 35(1972) 537-542.

[3] O’NEIL, B., Semi-Riemannian Geometry, Academic Pres, New York, London, 1983.

[4] THAS, C., Een (lokale) Studie van de $(m+1)$-dimensionale varieteiten, van de $n$-dimensionale Euclidische Ruimte $\mathbb{R}$ ( $n \geq 2 m+1$ en $m \geq 1$ ), Beschreven door een Eendimensionale Familie van m-dimensionale Lineaire Ruiten. Paleis Der Academien Hertogsstreet, I, Brussel, (1974).

[5] THAS, C., Properties of Ruled Surfaces in the Euclidean Space $E^{\mathrm{a}}$ Academia Sinica Vol 6, No.1, 133-142, 1978. 